\title{
A Transversalidade do Conhecimento da Saúde Coletiva no Currículo de Medicina de uma Escola Médica Pública: Relevância das Disciplinas na Formação dos Alunos
}

\author{
Crosscutting Knowledge in Collective Health \\ in a Public Medical School Curriculum: the \\ Relevance of Disciplines in Undergraduate \\ Medical Training
}

\author{
José Alberto Alves Oliveiral \\ Francisco Júlio Muniz Neto \\ Francisco José Maia Pinto ${ }^{I}$ \\ Marcelo Gurgel Carlos da Silval \\ Maria Salete Bessa Jorgel
}

PALAVRAS-CHAVE

- Educação Médica.

- Estudantes de Medicina.

- Currículo.

- Saúde Coletiva.

KEY-WORDS

- Education, Medical.

- Curriculum.

- Public Health.

Recebido em: 20/05/2009 Aprovado em: 27/10/2009

\section{RESUMO}

O curso de Medicina incorpora a transversalidade das disciplinas da saúde coletiva (Ciências Sociais e Humanas, Epidemiologia, Planejamento, Gestão e Avaliação dos Serviços e Programas de Saúde). Objetiva-se avaliar a formação do aluno de Medicina ante as disciplinas da saúde coletiva, descrever a integração das atividades desenvolvidas e analisar a importância dessas disciplinas segundo os discentes. Este é um estudo descritivo com abordagem quantitativa, realizado na Universidade Estadual do Ceará e em unidades de saúde conveniadas ao curso de Medicina da Uece. A amostra compôs-se por 129 alunos do curso de Medicina. Utilizou-se um questionário com a maioria das questões fechadas. A análise dos dados foi processada com o programa estatístico SPSS 16.0 para Windows, de forma descritiva e com medidas paramétricas, envolvendo média e desvio-padrão. A maioria dos estudantes $(67,1 \%)$ ressaltou a relevância das disciplinas da saúde coletiva no seu curso e as conceituou entre bastante importantes e muito importantes. Conclui-se que os estudantes consideram essencial a inserção das disciplinas no curso. Segundo revelado, a saúde coletiva é percebida como uma ferramenta fundamental no seu processo de construção do conhecimento.

\section{ABSTRACT}

Medical schools incorporate various crosscutting disciplines from collective health (social and human sciences, epidemiology, planning, and management and evaluation of health services and programs). This article aims to evaluate undergraduate medical training in relation to course subjects in collective (or public) health, describe the integration of the related activities, and analyze the importance of these disciplines from the students' perspective. This was a descriptive study with a quantitative approach at the State University of Ceará, Brazil, in health units operating in collaboration with the school of medicine. The sample consisted of 129 undergraduate medical students. A questionnaire was used, mainly with closed questions. Data analysis was performed with SPSS 16.0 for Windows, both descriptively and parametrically, involving means and standard deviations. The majority of the students (67.1\%) highlighted the relevance of disciplines in collective health during their course of study, classifying them as important or very important. The study concluded that the students consider the inclusion of such disciplines essential. Thus, collective or public health is perceived as a fundamental tool in the undergraduate knowledge-building process. 


\section{INTRODUÇÃO}

Ao longo do tempo, o ensino médico vem sofrendo modificações e sendo alvo do estudo de diversos pesquisadores, entre eles o educador Abraham Flexner (1866-1959), autor do relatório Flexner, que avaliou o ensino médico em instituições dos Estados Unidos e do Canadá. Esse estudo serviu para mudar os paradigmas do ensino médico até então vigentes ${ }^{1}$.

Durante todo o século passado, a formação médica se pautou na valorização do profissional especialista, com certa desvalorização da figura do médico generalista. Isto pode ser atribuído ao advento de novas tecnologias na parte diagnóstica e terapêutica, o que exige do médico dar resolutividade ao uso dessas tecnologias. O próprio ensino de graduação nas escolas, com a valorização da aprendizagem em centros de excelência em detrimento dos centros de atenção básica, favorece tal formação, pois em nível de atenção terciária o aluno se depara com situações muito complexas e específicas ${ }^{2}$.

Em virtude do quadro de não resolutividade desses profissionais egressos diante das necessidades da população, foram propostas várias mudanças no currículo das escolas médicas. Uma delas foi o incremento das Ciências Sociais e Humanas na grade curricular, para despertar no aluno a percepção do paciente não apenas quanto ao aspecto patológico da sua enfermidade, mas também para uma análise dos fatores biopsicossociais ${ }^{3}$.

Outra mudança decisiva foi a implantação da saúde coletiva nos currículos médicos. O conjunto de disciplinas componentes desse campo de conhecimento leva o aluno a desenvolver a ideia de multidisciplinaridade, com base na qual as disciplinas são vistas de forma integrada, todas num mesmo nível de valorização ${ }^{4}$.

Apesar dos esforços em torno da mudança do ensino médico para adequá-lo às necessidades da população, no intuito de capacitar o profissional egresso da graduação a resolver ou atenuar os problemas de saúde da população, é preciso transpor inúmeras barreiras no que se refere a tais mudanças. Esses empecilhos não residem somente na figura do aluno, nem sempre interessado nas disciplinas sociais; também o corpo docente apresenta limitações, sobretudo por não ter se adaptado a esse novo processo didático-pedagógico, algumas vezes, em determinados aspectos, contrário às práticas do corpo docente ${ }^{5}$.

Nesse contexto, os discentes perceberam a necessidade de avaliar as constantes mudanças acadêmicas concernentes à formação de profissionais médicos. Para isso, evidenciaram-se as falhas do modelo de ensino então vigente, que valorizava a gênese de um profissional nos moldes do taylorismo. Conforme exigido pelas novas propostas de educação, o processo de aprendizagem deve transcender os conhecimentos fisiopatológicos e adentrar as diversas relações sociais que se articulam em torno do processo saúde-doença com vistas a um melhor entendimento do fenômeno estudado.

Em sua estrutura, o curso de graduação em Medicina da Uece tem como eixo do desenvolvimento curricular as necessidades de saúde mais frequentes, referidas pela população e identificadas pelo setor saúde. Apesar disso, é sua intenção utilizar metodologias que privilegiem a participação ativa do discente na edificação do saber e a integração entre os conteúdos, além de incentivar a interação entre o ensino, a pesquisa e a extensão/assistência ${ }^{6}$.

No transcorrer dos semestres letivos, de acordo com a grade curricular, as disciplinas relacionadas à saúde coletiva enfatizarão o contato do aluno com a comunidade e, ao mesmo tempo, se estimulará sua participação em estágios na área de saúde comunitária e sua inclusão em programas de saúde da família, assim como em atividades de extensão junto a ONGs e outros aparatos sociais ${ }^{6}$.

Em virtude disso, pretende-se incluir dimensões éticas e humanísticas na grade curricular, de forma a estimular no estudante atitudes e valores orientados para a cidadania. De maneira semelhante, privilegiou-se o seguinte: promover a integração e a interdisciplinaridade, em coerência com o eixo de desenvolvimento curricular, na tentativa de integrar as dimensões biológicas, psicológicas, sociais e ambientais; inserir o aluno precocemente em atividades práticas relevantes para a sua futura vida profissional; utilizar diferentes cenários de ensino-aprendizagem para propiciar ao aluno conhecer e vivenciar situações variadas de vida, da organização da prática e do trabalho em equipe multiprofissional ${ }^{6}$.

Com esta finalidade, o perfil delineado para o médico egresso da instituição contempla: formação generalista, humanística, crítica e reflexiva, com vistas a capacitá-lo para atuar, com base em princípios éticos, no processo de saúde-doença nos diferentes níveis de atenção, desenvolvendo ações de promoção, prevenção, recuperação e reabilitação à saúde, na perspectiva da integralidade da assistência, como agente promotor da saúde integral do ser humano ${ }^{6}$.

\section{OBJETIVOS}

Este estudo visa, como principal objetivo, avaliar a formação do aluno de Medicina mediante a transversalidade das disciplinas da saúde coletiva (Ciências Sociais e Humanas, Epidemiologia, e Planejamento, Gestão e Avaliação dos Serviços e Programas de Saúde) do curso de Medicina da Universidade Estadual do Ceará (Uece). Os objetivos específicos foram: descrever a integração das atividades desenvolvidas 
ao longo das disciplinas da saúde coletiva, na concepção dos estudantes de Medicina, e analisar como estes se apropriam e percebem a importância das disciplinas da saúde coletiva e as contribuições destas para sua formação acadêmica.

\section{METODOLOGIA}

Trata-se de estudo transversal, descritivo e com abordagem quantitativa, desenvolvido nas seguintes instituições: Instituto Superior de Ciências Biomédicas (ISCB) da Universidade Estadual do Ceará (Uece) e unidades conveniadas com o curso de Medicina da referida instituição (Policlínica Sol Nascente, Hospital Geral César Cals, Hospital Infantil Albert Sabin e Hospital Geral de Fortaleza), de dezembro de 2008 a março de 2009.

A amostra foi composta por 129 alunos distribuídos do primeiro ao décimo primeiro semestre, aos quais se aplicaram os questionários. Os critérios de inclusão foram: estar regularmente matriculado no curso de Medicina da Uece de dezembro de 2008 a março de 2009 e ter cursado pelo menos uma das disciplinas do conjunto da saúde coletiva, isto é, alunos a partir do segundo semestre. Já o critério de exclusão da amostra foi este: estudantes que estavam desenvolvendo as atividades do internato fora de Fortaleza no período da pesquisa.

Todas as variáveis estudadas se encontram no questionário preenchido pelos alunos com perguntas sobre as características gerais dos estudantes (sexo, idade, semestre que cursa, procedência e naturalidade). Foram selecionadas as variáveis a seguir, que respondem aos objetivos específicos respectivos: (1) avaliação da importância das disciplinas da saúde coletiva no curso de Medicina da Uece e (2) características da formação dos alunos do curso de Medicina da Uece.

Os alunos preencheram o questionário com 17 questões, a maioria fechadas. Para estas havia três opções: sim, não e não sabe. Na primeira questão, utilizaram-se escores para classificar a conceituação dos alunos: (1) nada importante; (2) pouco importante; (3) moderadamente importante; (4) muito importante; (5) bastante importante. Do total, aproveitaram-se seis questões que respondiam aos objetivos deste estudo. Para as respostas aos questionários adotaram-se dois critérios. Os alunos com aula no ISCB responderam os questionários nos últimos minutos das aulas, mediante autorização do professor. No caso dos internos (alunos do quinto ano), os questionários foram entregues nos respectivos serviços nas unidades conveniadas onde eles se encontravam.

Os dados foram processados no programa estatístico Statistical Package for the Social Science - SPSS, versão 16.0, para Windows. Os dados gerais foram analisados de forma descritiva, usando-se as frequências simples e percentuais e também pelas medidas paramétricas, envolvendo a média e o desviopadrão. Posteriormente, encontrou-se o intervalo $\bar{X} \pm 2 S$, a fim de se identificar a quantidade de alunos fora do intervalo apresentado, no nível de significância de 5\%.

\section{RESULTADOS E DISCUSSÃO}

Dos 129 alunos que responderam ao questionário, a maioria, 66 (51,2\%), era do sexo masculino, com procedência de Fortaleza, 127 (98,4\%), tendo como medidas paramétricas aproximadas idade média de 22 anos e desvio-padrão de três anos. A idade mínima do grupo de estudo era de 18 anos e a máxima de 36 anos. Verificou-se, ainda, uma assimetria positiva de 1,22 , representada por $75 \%$ do grupo de estudo, a indicar maior concentração em idades abaixo de 24 anos. No nível de significância de $5 \%$, apenas seis alunos tinham idades fora do intervalo de confiança $\bar{X} \pm 2 S$ (22 anos \pm 3 anos).

Inicialmente, os discentes avaliaram a importância das disciplinas componentes da saúde coletiva no seu curso e as conceituaram em nada importantes, pouco importantes, moderadamente importantes, bastante importantes e muito importantes. No questionário, foi atribuída a numeração de 1 a 5 para a resposta (Tabela 1 ).

Tabela 1

Avaliação da importância das disciplinas da saúde coletiva no curso de Medicina, Uece, Fortaleza, dez. 2008/mar. 2009

\begin{tabular}{|c|c|c|}
\hline Importância no curso & Frequência & $\%$ \\
\hline Não responderam* & 7 & 5,4 \\
\hline 1 & 2 & 1,6 \\
\hline 2 & 9 & 7,0 \\
\hline 3 & 36 & 27,9 \\
\hline 4 & 55 & 42,6 \\
\hline 5 & 20 & 15,5 \\
\hline Total & 129 & 100,0 \\
\hline
\end{tabular}

*Respostas ordenadas de 1 - mínima a 5 - máxima.

Excluídos os sete alunos que deixaram de responder (5,4\%), a maior parte, $55(42,6 \%)$, referiu que as disciplinas são bastante importantes no curso. A maioria, $75(67,1 \%)$, conceituou as disciplinas entre bastante importantes e muito importantes.

Em estudo semelhante, Campos $^{7}$ avaliou a percepção dos alunos quanto ao estágio em saúde da família. Estes conceituaram como bastante importante (48\%) e muito importante $(27,5 \%)$ o estágio nas unidades de saúde da família vinculadas ao curso de Ciências Médicas da Faculdade de Medicina de Ribeirão Preto da Universidade de São Paulo. 
No que se refere ao questionamento sobre a contribuição das disciplinas na formação do médico, de acordo com a maioria, $112(86,8 \%)$, as disciplinas realmente contribuíam na formação do médico. Apenas 10 (7,8\%) não souberam opinar e $7(5,4 \%)$ responderam negativamente (Tabela 2$)$.

Tabela 2

Características da formação dos alunos do curso de Medicina, Uece, Fortaleza, dez. 2008/mar. 2009

\begin{tabular}{lrr}
\hline & $\mathrm{N}=129$ & $\%$ \\
\hline Contribuição na formação & 7 & 5,4 \\
$\quad$ Não & 10 & 7,8 \\
$\quad$ Não sabe & 112 & 86,8 \\
$\quad$ Sim & & \\
Inserção na grade curricular & 8 & 6,2 \\
$\quad$ Não & 4 & 3,1 \\
$\quad$ Não sabe & 117 & 90,7 \\
$\quad$ Sim & & \\
Docente - qualificação & 36 & 27,9 \\
$\quad$ Apenas alguns & 5 & 3,9 \\
$\quad$ Não & 88 & 68,2 \\
$\quad$ Sim & & \\
Aversão às disciplinas & 2 & 1,5 \\
$\quad$ Não responderam & 92 & 71,3 \\
$\quad$ Não & 6 & 4,7 \\
$\quad$ Não sabe & 29 & 22,5 \\
Sim & & \\
Abordagem de temas em saúde pública & & 13,2 \\
$\quad$ Não & 17 & 2,3 \\
$\quad$ Não sabe & 3 & 84,5 \\
Sim & 109 & \\
\hline
\end{tabular}

Quando indagados sobre a importância da inserção dessas disciplinas na grade curricular do seu curso, a grande maioria, 117 (90,7\%), respondeu afirmativamente, enquanto uma minoria, $8(6,2 \%)$, considerou como não sendo importante (Tabela 2).

Carvalho et al. ${ }^{8}$, ao avaliarem as experiências em torno do ensino da saúde coletiva na Faculdade de Ciências Médicas da Unicamp, comentam os discursos dos alunos quanto à contribuição da disciplina na formação profissional deles: "As falas dos alunos aqui reproduzidas indicam que algo caminhou: são discursos afetivos e afetados pelo complexo texto, rico e desafiador, do cotidiano das relações sociais".

Na Tabela 2, quanto à característica "inserção na grade curricular", os dados mostram que as novas diretrizes curriculares, propostas pelo MEC em 2001, com a inserção obrigatória da saúde coletiva nos currículos das escolas médicas vêm ten- do considerável aceitação dos estudantes de graduação. Como afirma Lampert ${ }^{9}$, o currículo não é um elemento estático, pois deve se adequar à organização da sociedade e da educação.

Na pergunta que avaliou se os alunos consideram que o corpo docente dessas disciplinas estava apto ou não para ministrar o conteúdo programático, 88 (68,2\%) responderam de forma positiva, porém para $36(27,9 \%)$ apenas parte do colegiado dessas disciplinas estava qualificado para ministrar os conteúdos (Tabela 2). Isso nos leva a refletir sobre a necessidade da educação permanente dos docentes envolvidos neste campo, no intuito de torná-los aptos a acompanhar o desabrochar de novas concepções e conhecimentos. Desse modo, será possível discutir historicamente a formação médica e de profissionais da equipe de saúde que se espelham em um modelo centrado na doença, pois o campo da saúde coletiva envolve saberes e práticas pautados na clínica, em que o sujeito é o centro das decisões. Este campo é, pois, dinâmico e desafiador.

Como ressaltam Ceccim e Feuerwerker ${ }^{10}$, o trabalho na área da saúde é um trabalho de escuta, porquanto a interação entre os diversos sujeitos envolvidos é de suma relevância na qualidade da resposta assistencial. Ademais, o incremento de novas tecnologias é constante e repercute na decisão e concretização "[...] da responsabilidade tecnocientífica, social e ética do cuidado, do tratamento ou do acompanhante em saúde".

Segundo Costa ${ }^{5}$, determinados fatores limitam mudanças na atuação docente em Medicina. Entre estes: "desvalorização das atividades de ensino e supremacia da pesquisa", por ser a docência vista como uma atividade em segundo plano na profissão médica, bem como haver mais valorização da pesquisa em detrimento da docência; "falta de profissionalização docente", pois a identidade profissional do professor não pode ser desvinculada da perspectiva da formação docente; "desvalorização da formação docente do professor de Medicina", uma vez que, embora não se deva desconsiderar a capacidade autodidata dos docentes, a construção do saber como resultado único da experiência é insuficiente; "resistência docente a mudanças", sobretudo porque as tentativas de mudanças curriculares trariam certa insegurança aos professores; "individualismo da ação docente", visto que a distribuição dos conteúdos entre os docentes resulta das pretensões e das preferências de cada um, não havendo uma discussão mais ampla dos métodos pedagógicos.

Inegavelmente, a formação não pode se assentar apenas na busca incessante pela etiologia, diagnóstico, tratamento e prognóstico das patologias e agravos. Urge atender às necessidades da população, da gestão setorial e do controle social em saúde, devendo-se objetivar a transformação das práticas e da própria organização do trabalho com vistas a propiciar acolhi- 
mento e cuidado às necessidades de saúde do indivíduo, do coletivo e das populações ${ }^{10}$.

Neste estudo, a pergunta "você tem alguma aversão pelas disciplinas do campo da saúde coletiva (Ciências Humanas, Sociais, Epidemiologia, Planejamento, Gestão e Avaliação em Saúde)?" foi respondida por $127(98,4 \%)$ do total de alunos. De acordo com o observado, a maioria destes, 92 (71,3\%), não demonstrou ter nenhuma antipatia em relação a essas disciplinas, enquanto $29(22,5 \%)$ responderam ter algum tipo de aversão. Parte destes, 21 (72,4\%), atribuiu a não empatia com as disciplinas da saúde coletiva principalmente à repetição desses conteúdos em disciplinas diferentes (Tabela 2).

Quando interrogados "se as atividades teóricas nessas disciplinas possibilitaram a abordagem de temas importantes em saúde pública", a maioria, 109 (84,5\%), respondeu de forma afirmativa. Isso mostra que as discussões de caso, seminários e aulas têm propiciado maior entendimento sobre conceitos importantes em saúde pública (Tabela 2).

Convém destacar a diferença entre saúde pública e saúde coletiva. Uma definição clássica para saúde pública é a de Terris ${ }^{11}$ : "a arte e a ciência de prevenir a doença e a incapacidade, prolongar a vida e promover a saúde física e mental mediante os esforços organizados da comunidade". Atualmente, porém, fala-se de uma "Nova Saúde Pública", que examina crítica e aprofundadamente os fatores determinantes da "crise da saúde pública". Já a saúde coletiva se caracteriza pela produção de conhecimentos sobre o processo saúde-doença, reunindo distintas disciplinas que o abordam sob diferentes ângulos. No entanto, no plano teórico-epidemiológico, a saúde coletiva aparece como um campo interdisciplinar. Desse modo, não se restringe apenas a uma especialidade médica ou disciplina científica ${ }^{12}$.

Com base nessa realidade, as atividades curriculares não devem ser desenvolvidas somente em sala de aula. Desde o início da sua formação, os alunos precisam aliar a prática às necessidades da população na atenção básica. Para Batista ${ }^{4}$, o aprendizado dos acadêmicos deve estar atrelado à prática e ao cotidiano em consonância com as necessidades e demandas da sociedade, "[...] respondendo aos desafios contemporâneos da ética, da integralidade, do cuidado e da intersetorialidade".(p.43)

Como ressaltam Machado et al. ${ }^{13}$, a integralidade requer um olhar mais amplo dos sujeitos envolvidos no processo saúde-doença, embora estes não sejam alcançáveis em sua totalidade, diante de todas as dimensões passíveis de intervenção. Em virtude disso, a prática do atendimento integral requer um compromisso mais firme entre a educação permanente e a prática multiprofissional, pois a formação dos trabalhadores de saúde deve transpor a barreira entre a atenção individual e a coletiva.

\section{CONCLUSÃO}

A maioria dos participantes desta pesquisa, $75(67,1 \%)$, ressaltou a elevada importância das disciplinas da saúde coletiva no seu curso e as conceituou entre bastante importantes e muito importantes. Cento e dezessete $(90,7 \%)$ concordaram com sua inserção na grade curricular, demonstrando empatia por esses conteúdos.

Segundo a concepção da maioria dos acadêmicos interrogados, 109 (84,5\%), as atividades desenvolvidas em sala de aula possibilitam a compreensão de assuntos relevantes em saúde pública. Verificou-se que as discussões de casos, as aulas teóricas e os seminários se mostraram eficazes para um melhor entendimento discente.

Em relação à forma como os alunos se apropriam das disciplinas e as percebem, a maioria dos discentes, 92 (71,3\%), não demonstrou nenhuma aversão aos conteúdos ministrados, porém uma parcela deles, 29 (22,5\%), revelou alguma antipatia em decorrência da repetição dos conteúdos em disciplinas diferentes.

Desde os semestres básicos até os mais avançados, os estudantes percebem e avaliam a inserção das disciplinas da saúde coletiva no currículo do curso como de suma importância no processo de formação dos futuros médicos. Como revelou a análise, os alunos veem na saúde coletiva uma ferramenta fundamental no seu processo de construção do saber.

\section{REFERÊNCIAS}

1. Kemp A, Edler FC. A Reforma Médica no Brasil e nos Estados Unidos: uma comparação entre duas retóricas. Hist Ciênc Saúde-Manguinhos. 2004;11(3):569-85.

2. Bulcão LC. O Ensino Médico e os Novos Cenários de Ensino-Aprendizagem. Rev Bras Educ Med. 2004;28(1):61-72.

3. Nunes ED. As Ciências Humanas e a Saúde: Algumas Considerações. Rev Bras Educ Med. 2003; 27(1):65-71.

4. Batista SHS. A Interdisciplinaridade no Ensino Médico. Rev Bras Educ Med. 2004;30(1):39-46.

5. Costa NMSC. Docência no Ensino Médico: por que é tão difícil mudar? Rev Bras Educ Med. 2007; 31(1):21-30.

6. Universidade Estadual do Ceará. Normas acadêmicas para o curso de Medicina. Fortaleza: Pró-Reitoria de Graduação. Centro de Ciências da Saúde; 2003.

7. Campos MAF. Percepção e avaliação dos alunos do Curso de Medicina de uma escola médica pública sobre a importância do estágio em saúde da família na sua formação. Rev Bras Educ Med. 2008;32(1):83-9.

8. Carvalho SR et al. O ensino da saúde coletiva no curso médico da Unicamp: experiências inovadoras junto às unidades básicas de saúde. Interface Comun.Saúde Educ. 2006;10(20):457-72 
9. Lampert JB. Tendências de mudanças na formação médica no Brasil. Rio de Janeiro; 2002. Doutorado [Tese] — Escola Nacional de Saúde Pública.

10. Ceccim RB, Feuerwerker LCM. O Quadrilátero da Formação para a Área da Saúde: Ensino, Gestão, Atenção e Controle Social. Physis. 2004;14(1):41-65.

11. Terris MT. Tendencias actuales em la salud publica de las Americas. In: Organización Panamericana de la Salud. La crisis de la salud pública: reflexiones para el debate. [S.1.]: OPS Publicación Científica; 1992. p.185-204.

12. Paim JS, Almeida Filho N. Saúde Coletiva: uma "nova saúde pública" ou campo aberto a novos paradigmas? Rev Saude Publica. 1998;32(4):299-316.

13. Machado MFAS et al. Integralidade, formação de saúde, educação em saúde e as propostas do SUS — uma revisão conceitual. Ciênc Saude Coletiva. 2007;12(2):335-42.

\section{CONTRIBUIÇÃO DOS AUTORES}

José Alberto Alves Oliveira participou da confecção do projeto de pesquisa que originou o artigo, da coleta de dados, da análise dos dados, da tabulação dos resultados, da revisão bibliográfica para discussão dos resultados e da correção ortográfica.
Francisco Júlio Muniz Neto, participou da coleta de dados, da tabulação dos resultados e da correção ortográfica do trabalho.

Francisco José Maia Pinto, participou da elaboração da metodologia cinetífica do trabalho, da análise dos dados e da tabulação dos resultados.

Marcelo Gurgel Carlos da Silva, participou d revisão do artigo em toda sua extensão e orientou a parte da introdução e objetivos do trabalho.

Maria Salete Bessa Jorge, participou da orientação de todo o trabalho desde a elaboração do projeto de pesquisa até a elaboração e revisão final do artigo em toda sua extensão.

\section{CONFLITOS DE INTERESSE}

Declarou não haver

\section{ENDEREÇO PARA CORRESPONDÊNCIA}

José Alberto Alves Oliveira

Rua Pintor Antônio Bandeir, 1751 - apto 102

Vicente Pinzon - Fortaleza

CEP 60182-296 - CE

E-mail: albertiezzi@hotmail.com 